\title{
An Analysis of Risk Communication Surrounding Increases in a Polio-like Condition in the U.S.
}

\author{
Amie Nisler \\ Northrop Grumman, Decatur, Georgia, United States \\ Objective
}

To assess the type, tone, consistency, and accuracy of communications surrounding a rare polio-like condition called acute flaccid myelitis between 2014-2017 from from CDC, other health agencies, researchers, news media outlets, and the public.

\section{Introduction}

In 2014, CDC started receiving an increase in reports of children in the United States with unexplained limb weakness or paralysis (120 total cases). These children were later confirmed by neurology experts to have a rare condition called acute flaccid myelitis (AFM). The Council of State and Territorial Epidemiologists created a standardized case definition for AFM in 2015, allowing CDC to establish standardized surveillance to monitor AFM, determine possible causes and risk factors, and attempt to estimate the baseline incidence. Through this surveillance, CDC identified another increase in AFM cases in 2016 (149 total cases), and obtained valuable information on the clinical presentation to help characterize this illness and the epidemiology of AFM. However, despite the ongoing investigation, many questions still remain about AFM, including why the increases occurred and what has caused most of the AFM cases. The lack of AFM knowledge has made preventing AFM, finding effective treatments for patients, and developing communication messages challenging.

\section{Methods}

We compiled a timeline of events surrounding AFM and the investigation from 2014 to 2017, and across this timeframe, we analyzed communications from CDC, other health agencies, researchers, news media outlets, and the public. We reviewed scientific articles, press releases, websites, social media, educational materials, and news stories. We assessed the type, tone, consis tency, and accuracy of the AFM information based on the principles in CDC's Crisis and Emergency Risk Communication Manual.

\section{Results}

The AFM communications included information about possible causes, symptoms, severity, transmission, risk, prevention, prognosis, and the possibility of future increases. Several materials included stories about patients. Information from the different sources evolved along with the investigation and was overall consistent, but especially differed on whether AFM was associated with enterovirus D68. The amount of information released from the different sources was also variable, with some sources releasing more information than others.

\section{Conclusions}

Emerging diseases, like AFM, pose threats to the public's health, requiring credible and timely risk communication so people can make decisions about their well-being. CDC has played a critical role in relaying the best available scientific information about AFM in a timely manner to healthcare professionals and the public, as the situation has evolved since 2014 and there have been many unknowns. The messages we communicate during these times, using risk communication principles and the valuable data collected from our AFM surveillance and investigation, and their timing affects our level of involvement in the national conversation about AFM. Working closely with health departments, healthcare providers, researchers, and other partners is important for consistent communication messaging and release of information. 